\title{
Faecal carriage of klebsiella by patients with ankylosing spondylitis and rheumatoid arthritis
}

\author{
R. E. WARREN ${ }^{1}$ AND D. A. BREWERTON ${ }^{2}$
}

From the ${ }^{1}$ Clinical Microbiology and Public Health Laboratory, Addenbrooke's Hospital, Cambridge, and the ${ }^{2}$ Department of Rheumatology, Westminster Hospital, London

SUMMARY In consecutive samples submitted to a clinical microbiology laboratory 22 out of 99 from outpatients and 23 out of 51 from inpatients yielded Klebsiella sp. A clinical reassessment of outpatients with ankylosing spondylitis (AS) and rheumatoid arthritis (RA) who had not been inpatients within the last year was made for disease astivity and drug requirements. 124 patients with AS and 92 with RA were requested at assessment to submit a stool specimen for klebsiella examination, this being carried out without disclosure of the patient's clinical category. Two months later a questionnaire on symptom changes was collected and the results correlated with klebsiella carriage. Eighty-nine patients with AS and 82 patients with RA fulfilled all criteria for assessment. Of those assessed, 24 out of 89 AS patients and 26 out of 82 RA patients had klebsiella in the faeces. There was no correlation between the initial clinical assessment category and klebsiella carriage. Seventy patients with AS and 57 patients with RA had no change in symptoms over the 2-month period. Nineteen AS patients and 31 RA patients noted symptom improvement or worsening. Of these, 3 AS and 10 RA patients had klebsiella in their faeces. There was no correlation between worsening of symptoms over a 2-month period and klebsiella carriage at initial assessment.

Peripheral, sterile, 'reactive' arthritis following enteric infection with Yersinia enterocolitica (serotypes 3 and 9 of the 34 serotypes) (Ahvonen et al., 1969), Shigella sp. (especially Sh. flexneri) (Good and Schultz, 1977), and Salmonella sp. (especially $S$. typhimurium) (Vartainen and Hurri, 1964; Warren, 1970 ) is associated with possesion of the histocompatibility antigen HLA B27. This has prompted speculation that acute anterior uveitis or ankylosing spondylitis, which are also associated with HLA B27, might be precipitated by asymptomatic colonisation of the gut by, or infection with, an organism carrying an antigen common to all these genera. This organism or a group of such organisms might be considered conventionally by microbiologists to be a member of the commensal flora rather than a pathogen. The importance of describing such an organism is that colonisation, and hence environmental provocation of an otherwise genetically determined disease, might be preventable regardless of the immunological mechanism involved in producing the clinical manifestations.

Accepted for publication 9 March 1979

Correspondence to Dr D. A. Brewerton, Department of Rheumatology, Westminster Hospital, Dean Ryle Street, Horseferry Road, London SW1P 2AP.
In 1976 Ebringer and his colleagues reported at a meeting under the auspices of INSERM that, as a result of a prospective search, they had found that Klebsiella aerogenes (a species of a genus in the tribe Klebsielleae of the Enterobacteriacae) shared antigens with HLA B27 lymphocytes when tested with rabbit antisera (Ebringer et al., 1976). The same group subsequently reported a cross-reactivity with Enterobacter aerogenes, a different genus in the same tribe, and Yersinia enterocolitica, another member of the Enterobacteriacae (Ebringer et al., 1977).

No detailed description of the experiments establishing the serological cross-reactions has to our knowledge been published or referred to in the extensive comment this abstract excited. Klebsiella pneumoniae (Edwards and Ewing, 1972) has been described in further work as the Klebsiella species responsible (Ebringer et al., 1978). This apparent variation in the implicated species is in fact illusory and due to the differences in British and American nomenclature. The USA species Klebsiella pneumoniae (Edwards and Ewing, 1972) includes a wide variety of species biochemically subdivided in the UK into $K$. aerogenes, $K$. pneumoniae (sensu strictu), $K$. edwardsii, and $K$. atlantae (Cowan and Steel, $1965)$ and serologically contains some 75 capsular 
serotypes. A majority of the $K$. pneumoniae strains (Edwards and Ewing, 1972) implicated by Ebringer et al. (1977) appear to be K. aerogenes. Klebsiella species are common agents in infections acquired in hospitals in the UK. For example, they are the second commonest cause of confirmed Gramnegative bacteraemia after Escherichia coli (Communicable Disease Report, 1977). Klebsiella spp. have been isolated from up to $30 \%$ of stools examined in those just admitted to hospital and those who are inpatients for some time (Casewell and Phillips, 1978a; Datta, 1969; Selden et al., 1971). Ebringer and his colleagues have recently claimed that carriage of $K$. pneumoniae (Edwards and Ewing, 1972) or Enterobacter aerogenes in the gut precedes a change in symptoms in patients with B27 (Ebringer et al., 1978).

Biochemical bacterial taxonomy may not be the best way of describing how man in immunological terms sees his commensal flora. Antigenic crossreactions are common between bacteria in closely related genera, but their systematic study has not been pursued except as an explanation of observations made in the course of development of serotyping methods.

No antigenic cross-reaction has to our knowledge been reported between Klebsiella, Enterobacter, and strains of Salmonella, Shigella, Yersinia, or Chlam$y$ dia isolated from patients with HLA B27 who have developed peripheral arthritis after infection with such strains. However, many serological crossreactivities are known between these genera, Escherichia coli, and Brucella-another species which has been reported as a provocative factor for spondylitis in persons with HLA B27 (Hodinka et al., 1978).

Yersinia pseudotuberculosis O serotypes crossreact with Salmonella $O$ serotypes because they share common 3-6 dideoxyhexose epitopes (Davies, 1958, 1961). Enterobacter cloacae and Escherichia coli serotypes 017 and 077 cross-react with $Y$. pseudotuberculosis serotype IVA (Knapp, 1968). Yersinia pseudotuberculosis is not known to provoke reactive arthritis more commonly in individuals with HLA B27, though a small number of cases have been recorded (Ahvonen, 1972; Chambers et al., 1978).

Yersinia enterocolitica serotype 9 is known to cross-react with Vibrio cholerae (Barua and Watanabe, 1972), and $O$ antigenic relationships between Salmonella, $V$. cholerae, and Escherichia coli are described (Winkle et al., 1972). Yersinia enterocolitica serotype 012 cross-reacts with Salmonella O antigen 047 (Wauters et al., 1971). Yersinia enterocolitica serotype 9 cross-reacts with Brucella abortus, B. melitensis, and B. suis (Hurvell et al., 1971). B. abortus cross-reacts with Vibro cholerae (Feeley, 1969).

Many $\mathbf{O}$ antigenic cross-reactions of Escherichia and Shigella spp. have been described, including a cross-reaction between Sh. flexneri types $4 \mathrm{~b}$ and 5, and, respectively, E. coli 055 and 0129 (Edwards and Ewing, 1972).

At least 16 cross-reactivities between $E$. coli and Salmonella $\mathrm{O}$ antigens have been described (Orskov et al., 1977); and a further group of 4 cross-reactions between $E$. coli serotypes and $K$. pneumoniae (Edwards and Ewing, 1972) $O$ types are described (Kaufman, 1949; Orskov, 1954). No single crossreactivity between $\mathrm{O}$ antigens of Klebsiella, Shigella, and Salmonella in respect of their cross-reacting $E$. coli types is known.

Cross-reactivity of the acid polysaccharide capsules of $E$. coli ( $\mathrm{K}$ antigens) is currently under investigation in many laboratories These $\mathrm{K}$ antigens are different from the $\mathrm{K}$ antigens that are responsible for mucosal adherence in enteropathogenic infections and which seem to be proteins.

Many Enterobacteriacae produce a slime or $\mathbf{M}$ antigen. This cross-reacts with the $\mathrm{K} 30$ and $\mathrm{K} 39$ antigens of $E$. coli and the K8, K11, K13, K21 and K35 antigens of Klebsiella (Henriksen, 1954). An additional antigen, the common enterobacterial antigen of Kunin, is present, even if not expressed in immunogenic form, in many Enterobacteriacae, including Salmonella typhimurium and Yersinia enterocolitica serotypes 3 and 9 (Maeland and Digranes, 1975).

The above brief survey indicates some of the very complex antigenic relationships, even within well defined species and classification serotypes, of the genera under discussion as provocative agents in individuals with HLA B27. Clearly another antigen not used in these serotyping schemes might actually be the hypothetical common antigen.

The potential importance of the reported association of prior carriage of Klebsiella pneumoniae (Edwards and Ewing, 1972) or Enterobacter aerogenes with subsequent change in symptoms, in patients with B27, encouraged us to conduct a further comparable study of patients with active and inactive AS using patients with RA as controls. A number of faeces submitted to a clinical microbiology laboratory for other reasons were also examined as a control on the bacteriological procedures used to isolate klebsiella, since no standard method for this has to our knowledge been published.

\section{Patients and methods}

CLINICAL S T UDY

During the period 17 March to 2 June, 1978, 124 
patients with ankylosing spondylitis (AS) and 92 with rheumatoid arthritis (RA) were reassessed by one observer (DAB). All were outpatients attending the Rheumatology Department, Westminster Hospital, and had not been inpatients in the past year. They fulfilled the New York criteria for AS and the ARA criteria for definite RA, and in no case was the diagnosis in doubt. Patients with psoriasis, chronic inflammatory bowel disease, or Reiter's disease, non-Caucasians and all first generation immigrants of any race, were excluded. Those AS and RA patients included in the study were seen in approximately equal numbers throughout fortnightly periods of the study.

Each patient was clinically assessed as having quiescent disease requiring no anti-inflammatory drugs, or apparently active disease that was well controlled by drugs, or clearly active inflammatory disease of spine, peripheral joints, or eyes.

On clinical grounds the activity of disease in the spine or peripheral joints was recorded as mild, moderate or severe. Acute episodes of inflammatory disease of spine, peripheral joints or eyes in the preceding 2 months were recorded. Also noted were the date of the last hospital attendance, the last consumption of hospital food or drink, all drug therapy (including antibiotics) within the past year, and a history of hydrotherapy. The patient was asked if his rheumatic or eye symptoms had ever been clearly better or worse following an infection such as a cold, bronchitis, diarrhoea, abdominal upset, or urinary infection, or during the 6 weeks following such an infection. It was noted if the patient had a history of uveitis or peripheral arthritis in the case of AS, or possession of serum rheumatoid factor or erosive changes if classified as RA. The patient was asked to post to one of us (REW) a specimen of the next stool passed, the stool being identifiable only by a number, whose clinical correlates were not disclosed until all cultures had been concluded. A questionnaire was explained to the patient (Table 1),

\section{Table 1 Questionnaire given to patients}

Remembering how much trouble you have had in the past two months, please return this form on $\ldots \ldots \ldots \ldots \ldots \ldots \ldots \ldots \ldots \ldots \ldots \ldots \ldots \ldots$ reporting your symptoms in the next two months from now. Do not mention minor changes in your symptoms.

Overall have you had:

Definitely less pain?

About the same?

Definitely more pain?

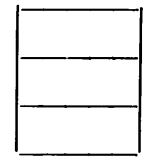

(please tick appropriate box)

If you have had more pain, please make a further appointment. If this is impossible or inconvient please write details on this form, including the nature of the pain, the date it occurred and whether you changed your drugs because of the pain. who was asked to complete and return it 2 months after assessment.

Forty-five patients entering the study were excluded from the analysis. One or more of the following justified exclusion: (a) no identifiable numbered faeces received; $(b)$ no identifiable postal questionnaire received; (c) stool sample received more than 14 days after initial assessment; $(d)$ questionnaire received less than 2 months or more than 3 months after receipt of faeces. Exclusions $(c)$ and $(d)$ were introduced to ensure a fixed time relationship between clinical assessments and bacteriological examination of the stool. Details of the 10 patients with RA and 35 with AS excluded are given in Tables 2 and 3 . These patients did not differ significantly from those included in their initial assessment category, history, age, sex, or culture results (where available).

BACTERIOLOGY AND CONTROLS

Cultures were carried out in the Clinical Microbiology Laboratory at Addenbrooke's Hospital,

Table 2 Comparisons of AS patients excluded from or included in assessment

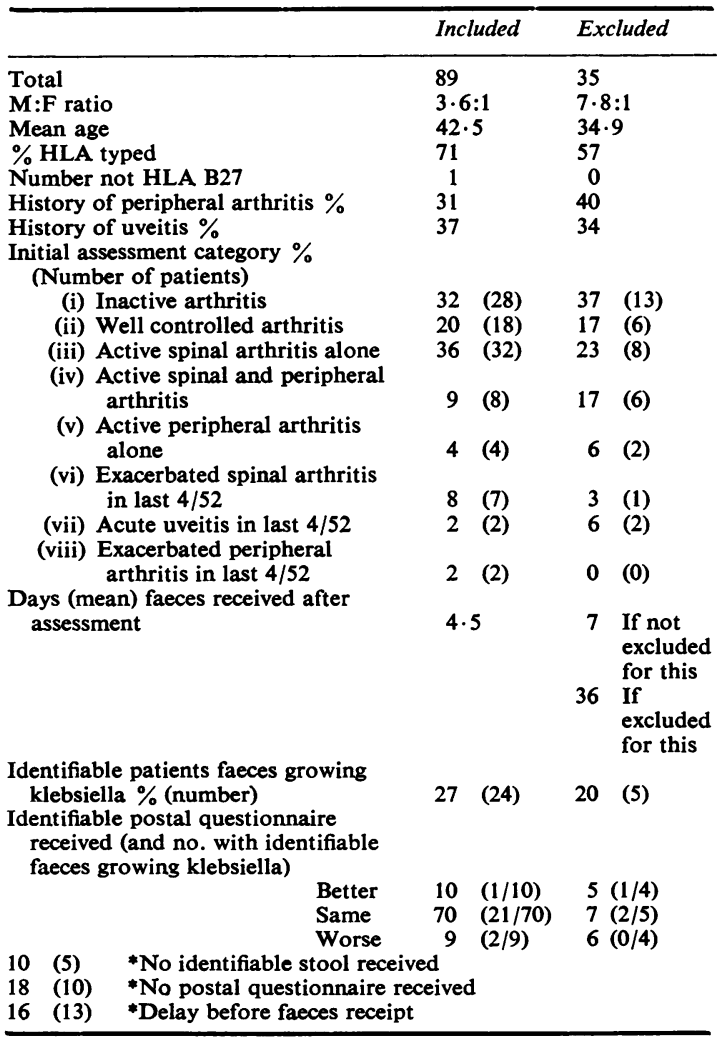

*Number excluded for each reason (bracketed figures are these where this was the sole reason for exclusion). 
Table 3 Comparison of RA patients excluded from or included in assessment

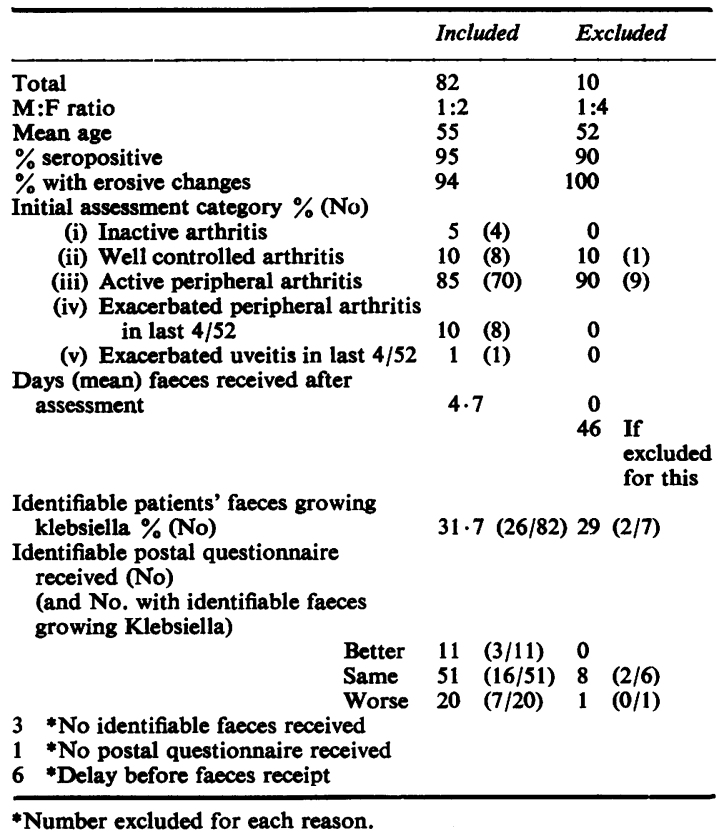

both directly and by enrichment. Direct cultures were made on plates of MacConkey base (Oxoid CM 7b) to which had been added $5 \% \mathrm{v} / \mathrm{v}$ of $20 \%$ inositol, $1 \% \mathrm{v} / \mathrm{v}$ of neutral red, and $10 \mathrm{mg} / \mathrm{l}$ carbenicillin. Plates were stored for up to 14 days at $+4^{\circ} \mathrm{C}$ before use. Plates were examined for inositol fermenting colonies after 18 hours at $36^{\circ} \mathrm{C}$. Enrichment cultures were made by emulsifying a pea-sized piece of faeces in bacteriological peptone (Oxoid) containing $10 \mathrm{mg} / 1$ ampicillin, incubating at $36^{\circ} \mathrm{C}$ for 18 hours, and subculturing to inositol carbenicillin MacConkey plates. Inositol fermenting colonies were tested in Donovan's medium for motility and inositol fermentation, in Simmonds's citrate medium, and PPA medium. Oxidase-negative, non-motile, inositol-fermenting, citrate-utilising strains that did not deaminate phenylalanine were accepted as Klebsiella spp. These strains from patients were kindly serotyped, phage-typed, and biotyped by Dr P. Mortimer, Public Health Laboratory Service Area Laboratory, Coventry, and confirmed as lysine decarboxylase positive, urease positive, gluconate positive, lactose fermenters, producing gas from glucose.

Enterobacter species were not sought, as these were found on only 9 out of 187 'positive' cultures from 163 patients by Ebringer et al. (1978).

212 consecutive faecal samples submitted to the Clinical Mticrobiology Laboratory in Feb.-March
1978 and 114 submitted in August and September 1978 were cultured by this method. Faeces submitted from patients attending general practitioner clinics or hospital inpatients were examined in February and March and from general practitioner clinics in August and September. These specimens had been submitted to investigate a history of diarrhoea or vomiting or to screen normal people for enteric pathogens, prior to employment as caterers, or because they were family contacts of patients with enteric pathogens. All faeces yielding enteric pathogens or from patients cultured previously were excluded from analysis.

A Klebsiella sp. was isolated in 22 out of 99 samples $(22 \%)$ from general practice in February and March, in 45 out of 114 samples (39\%) from general practice in August and September, and in 23 out of 51 samples (45\%) from Addenbrooke's Hospital inpatients during February and March. In addition, during this period faeces from patients in a geriatric ward at Huntingdon Hospital, where cases of salmonella infection had occurred, were examined repeatedly. Seven out of $33(21 \%)$ yielded klebsiella on first examination. Of the faeces of 29 patients examined on a second occasion 25 were repeatedly negative, 2 repeatedly positive, and 2 changed status.

\section{Results}

The average time since last outpatient attendance at hospital was 13 weeks for RA patients and 45 weeks for AS patients; this did not differ if the patient's faeces did or did not yield klebsiella. $39 \%$ of RA patients and $14 \%$ of AS patients with positive cultures had eaten hospital-produced food in the last year compared with $30 \%$ of RA patients and $15 \%$ of AS patients with negative cultures. $23 \%$ of RA patients and $41 \%$ of AS patients with positive cultures, and $19 \%$ of RA patients and $34 \%$ of AS patients with negative cultures, had received antibiotics in the last year. The klebsiella carriage rate was the same in the patients regardless of their antibiotic history. There was no correlation between exposure to other drugs and klebsiella carriage. Only 2 patients with positive cultures and 7 patients with negative cultures had received hydrotherapy. The known average duration of disease in patients with negative and positive cultures was the same, being respectively 19 and 10 years in AS and RA patients. In patients who were excluded the average disease duration was 15 years in both RA and AS patients.

Of those assessed, 24 out of 89 AS patients and 26 out of $82 \mathrm{RA}$ patients had klebsiella in their faeces. The klebsiella isolation rate in the 2 groups 
was comparable throughout the period of the study, rising from $12 \%$ ( 5 out of 42 ) in the first month to a constant $34 \%$ (45 out of 129) thereafter. Since patients were seen with active and inactive AS and RA and included in assessment at an equal rate throughout the study period, this change, which might reflect an undetected methodological variation or a seasonal change in carriage rate, does not affect the interpretation of results.

As tables 4 and 5 show, there was no correlation between the initial clinical assessment category and klebsiella carriage. Seventy patients with AS and 51 patients with RA reported no significant differences in pain due to their arthritis over the 2-month period. As Tables 6 and 7 show, there was no significant correlation between worsening of symptoms over a 2-month period and klebsiella carriage at initial assessment. $8 \cdot 3 \%$ of AS patients initially carrying klebsiella complained of worsening of symptoms compared with $10.8 \%$ of those not carrying klebsiella. $27 \%$ of RA patients initially carrying klebsiella complained of worsening of symptoms compared with $23 \%$ of those not carrying klebsiella.

Table 4 Klebsiella carriage and initial assessment in $A S$ patients

\begin{tabular}{|c|c|c|c|c|}
\hline \multirow[b]{3}{*}{ Inactive arthritis } & \multicolumn{3}{|c|}{$\begin{array}{l}\text { Number } \\
\text { (\% group) }\end{array}$} & \multirow[t]{2}{*}{$\begin{array}{l}\text { Total } \\
\text { number }\end{array}$} \\
\hline & \multicolumn{2}{|c|}{$\begin{array}{l}\text { Klebsiella } \\
\text { positive }\end{array}$} & \multirow{3}{*}{$\begin{array}{l}\begin{array}{l}\text { Klebsiella } \\
\text { negative }\end{array} \\
17 \\
15\end{array}$} & \\
\hline & 11 & (39) & & 28 \\
\hline $\begin{array}{l}\text { Well controlled arthritis } \\
\text { Active spinal arthritis }\end{array}$ & 3 & (7) & & 18 \\
\hline $\begin{array}{l}\text { alone } \\
\text { Active spinal and }\end{array}$ & 8 & (25) & 24 & 32 \\
\hline $\begin{array}{l}\text { peripheral arthritis } \\
\text { Active peripheral }\end{array}$ & 2 & (25) & 6 & 8 \\
\hline $\begin{array}{l}\text { arthritis alone } \\
\text { Exacerbated spinal }\end{array}$ & 0 & (0) & 4 & 4 \\
\hline arthritis in last $4 / 52$ & 1 & (14) & 6 & 7 \\
\hline $\begin{array}{l}\text { Acute uveitis in last } 4 / 52 \\
\text { Exacerbated peripheral }\end{array}$ & 1 & (50) & 1 & 2 \\
\hline $\begin{array}{l}\text { arthritis in last } 4 / 52 \\
\text { Total }\end{array}$ & $\begin{array}{r}0 \\
24\end{array}$ & $\begin{array}{l}(0) \\
(27)\end{array}$ & $\begin{array}{l}2^{*} \\
65\end{array}$ & $\begin{array}{r}2 \\
89\end{array}$ \\
\hline
\end{tabular}

*Plus 1 associated with rubella.

Table 5 Klebsiella carriage and initial assessment in $R A$ patients

\begin{tabular}{|c|c|c|c|c|}
\hline \multirow[b]{3}{*}{ Inactive arthritis } & \multicolumn{2}{|c|}{$\begin{array}{l}\text { Number } \\
\text { (\% group) }\end{array}$} & \multirow{2}{*}{$\begin{array}{l}\text { Number } \\
\begin{array}{l}\text { Klebsiella } \\
\text { negative }\end{array}\end{array}$} & \multirow[t]{2}{*}{$\begin{array}{l}\text { Total } \\
\text { number }\end{array}$} \\
\hline & \multicolumn{2}{|c|}{$\begin{array}{l}\text { Klebsiella } \\
\text { positive }\end{array}$} & & \\
\hline & 0 & $(0)$ & 4 & 4 \\
\hline $\begin{array}{l}\text { Well controlled arthritis } \\
\text { Active peripheral }\end{array}$ & 3 & (38) & 5 & 8 \\
\hline $\begin{array}{l}\text { arthritis } \\
\text { Exacerbated peripheral }\end{array}$ & 23 & (29) & 47 & 70 \\
\hline $\begin{array}{r}\text { arthritis in last } 4 / 52 \\
\text { Exacerbated uveitis in }\end{array}$ & 1 & (13) & 7 & 8 \\
\hline last $4 / 52$ & 1 & $(100)$ & 0 & 1 \\
\hline Total & 26 & (32) & 56 & 82 \\
\hline
\end{tabular}

Table 6 Association between pain changes in subsequent 2 months and initial klebsiella carriage in $A S$

\begin{tabular}{lcc}
\hline & $\begin{array}{l}\text { Number } \\
\text { initially } \\
\text { klebsiella } \\
\text { culture positive }\end{array}$ & $\begin{array}{l}\text { Number } \\
\text { initially } \\
\text { klebsiella } \\
\text { culture negative }\end{array}$ \\
\hline Symptoms better & 1 & 9 \\
Symptoms the same & 21 & $49^{*}$ \\
Symptoms worse & 2 & 7 \\
Total & 24 & 65 \\
\% symptoms worse & $8.3 \%$ & $10.8 \%$ \\
\hline
\end{tabular}

*1 patient in this group developed acute uveitis

Table 7 Association between pain changes in subsequent two months and initial klebsiella carriage in $R A$

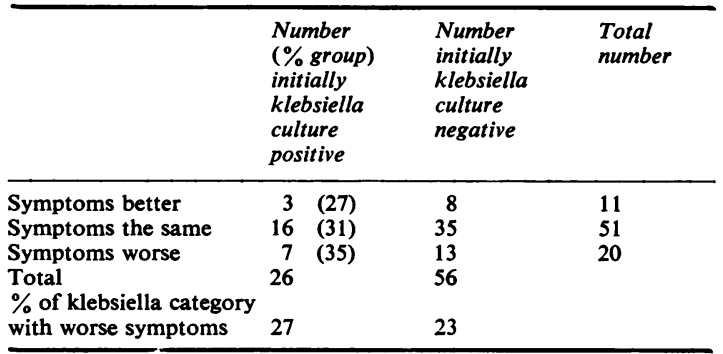

All three spondylitic patients with initial positive klebsiella cultures who commented on symptom change ( 2 worsening and 1 improvement) had active spinal disease. Of those patients with negative klebsiella cultures and symptom improvement, 2 had inactive, 2 well controlled, and 3 active spinal disease, and 1 had active spinal and peripheral arthritis. Of those with negative klebsiella cultures and symptom worsening, 1 had inactive, 2 well controlled, and 2 active spinal disease, and 2 had both active spinal disease and peripheral arthritis.

Assessing over a 4 month period (that is, 2 months before assessment and 2 months after assessment), klebsiella was present at initial assessment in 11 out of 48 of those who had either active disease of spine or peripheral joints, or a recent exacerbation of spine, joint, or eye disease, and in 13 out of 41 whose disease was either inactive or well controlled by drugs and who had no worsening of symptoms.

If those with the disease well controlled by drugs were included in the 'active' group, klebsiella was carried by 14 out of 63 with 'active' and 10 out of 26 with inactive disease. None of these differences is statistically significant.

None of the 11 patients with inactive disease from whom klebsiella was isolated or initial culture developed worsening of pain in the following 2 months. One out of the 17 patients with inactive disease in whom klebsiella was not found developed worsening of pain in the following 2 months. 
Table 8 Serotypes of klebsiella in RA and AS patients

Serotypes found in AS

$3,4,7(\times 2), 11,18,20,23,25,26,39,53(\times 4), 55,57,58,60,71$.

Non-typable $(\times 2)$

Serotypes found in RA

$3(\times 2), 8,9,10,14,18(\times 3), 19,23,27,35,38(\times 2), 47,53(\times 3), 55,59$

Non-typable $(\times 4)$.

Six out of 89 AS patients commented on past exacerbation of their symptoms after diarrhoea ( 3 culture-positive, 3 culture-negative) and 6 patients after upper respiratory tract infections ( 2 culturepositive, 4 culture-negative). Four AS patients had felt better during upper respiratory tract infections. Three out of $82 \mathrm{RA}$ patients complained of worse pain after diarrhoea (1 culture-positive, 2 culture-negative) and 8 patients after upper respiratory tract infections (3 culture-positive, 5 culture-negative).

Twenty-two out of 24 klebsiella isolates from AS patients and 22 out of 26 from RA patients were serotypable. Serotypes were randomly distributed between patients with AS and RA (Table 8). Twenty out of 22 from AS patients were Klebsiella aerogenes, of which 3 were indole producers and dulcitol fermenters. One serotype 23 and one serotype 58 were methyl red positive and were not $K$. aerogenes. Eighteen out of 23 from RA patients were $K$. aerogenes, of which 6 were indole producers and dulcitol fermenters. One serotype 23 , one type 38 , one type 10 , one type 3 , and one untypable were methyl red positive.

\section{Discussion}

Our results conflict with those reported by Ebringer and his colleagues in 4 respects. We found no higher isolation rate of klebsiella in patients with active spondylitis and no lower rate in patients with inactive diseases when compared with patients with rheumatoid arthritis attending the same clinic or with patients submitting faeces to the laboratory other than for rheumatological reasons. When single specimens of faeces were examined during 4-month periods, disease activity assessed over those periods was not related to faecal klebsiella carriage. Patients with inactive disease and positive cultures were no more likely to develop a disease exacerbation than those with inactive disease and negative cultures. We found that very few patients with spondylitis had learned to associate intercurrent infections (including diarrhoea or abdominal discomfort) with subsequent worsening of their rheumatic symptoms.

In several respects our study differs from those previously described. The numbers were lower because we excluded many patients-recent inpatients, those with associated diseases, non-Caucasians, and first generation immigrants. We also completed the study in a limited time, with approximately equal numbers of AS and RA patients in each week, both measures being designed to minimise changes in isolation rate due to methodological variation or seasonal change. Klebsiella is known to be present in cold rather than hot foods (Casewell and Phillips, 1978b). We did not exclude patients on the grounds of age over 40 years; there was no difference in klebsiella carriage rate in our patients aged under 40 , nor were they more likely to suffer disease exacerbations if culture-positive.

The present study may differ in important microbiological respects: faeces from AS patients, RA patients, and controls were examined only once. There is little information on the stability of klebsiella carriage in the human faecal flora (Montgomerie et al., 1970, Casewell and Phillips, 1978a). However, these reports, our limited studies in geriatric patients, and experience of other authors with Escherichia coli suggest that unusual frequency of sampling of active and inactive disease could distort the apparent frequency of klebsiella carriage if 1 positive out of a number of cultures is considered to imply positivity throughout a 3-month period. Hartley and her colleagues studied carriage of $E$. coli in 13 persons over a minimum period of 34 days (range 6-14 stools per individual) (Hartley et al., 1977). Within this period 10 of the majority of coliform colonies from 127 stools were examined and identified. These 13 people experienced 74 serotypes of $E$. coli. Nine serotypes were present for more than 14 days continuously; 24 serotypes were isolated on a single occasion only; 10 serotypes were isolated more than once but never on 2 consecutive occasions. On only 33 of 127 occasions were more than $2 E$. coli serotypes detected. Thus, repetitive sampling is likely greatly to modify detection of individual serotypes, let alone different intraserotype strains. The flora is relatively unstable when studied every 3 days in a rigorously standardised way.

We were unable to detect any relationship between patients with positive cultures and recent outpatient attendance, exposure to hospital food, hydrotherapy, antibiotics, or other drug therapy. Serotyping of our strains excluded any hospital acquired common source infection as a reason for a high carriage subgroup in patients with either AS or RA.

Thirty-eight out of 45 of our Klebsiella spp. were identified as Klebsiella aerogenes compared with 49 out of 99 in the earlier study (Ebringer et al., 1977), suggesting a non-random difference in Klebsiella spp. in the 2 studies. Serotyping would determine whether the disease exacerbations reported by 
Ebringer were caused by a particular serotype, with other serotypes having little clinical effect as we have found in our study. Such a finding would imply some common source producing colonisation. It would not necessarily imply that the serotype antigen itself was involved in disease production. It would, however, imply that some antigen on this particular strain was implicated. If this was found, it would then be possible to raise preimmunisation and postimmunisation sera against strains of the serotype associated with clinical exacerbations, and to test these sera in cross-absorption studies with HLA B27 lymphocytes. Until the details in such evidence-or other evidence-have been reported and confirmed, it will remain difficult to accept that Klebsiella spp. are clinically associated with disease activity in AS, or that the hypothesis of molecular mimicry explains the apparent association observed by Ebringer.

The authors gratefully acknowledge skilled technical assistance from Mr M. Amphlett, Mrs L. Wilcox, Mr M. Keys, and Mrs B. Walters.

Addendum. When this paper was presented to the Heberden Society on 24 November, 1978 Dr C. J. Eastmond, of Leeds, suggested that klebsiella carriage might be associated with the occurrence of attacks of acute anterior uveitis in patients with ankylosing spondylitis, even if it did not appear to be associated with disease activity in the spine or peripheral joints. This suggestion cannot be answered by the present report of a controlled study conducted in a limited period. However, we can record that over the past 2 years we have investigated 52 patients during attacks of acute anterior uveitis seen an average of 15 days after the onset of symptoms. Klebsiella spp. were present in 6 out of 29 with no associated disease and in 4 out of 19 with ankylosing spondylitis. The remaining 4 patients had Reiter's disease, RA, or spondylitis associated with chronic inflammatory bowel disease.

\section{References}

Ahvonen, P., Sievers, K., and Aho, K. (1969). Arthritis associated with Yersinia enterocolitica infection. Acta Rheumatologica Scandinavira, 15, 232-253.

Ahvonen, P. (1972). Human yersiniosis in Finland. I. Bacteriology and serology. Annals of Clinical Research, 4, 30-48.

Barua, D., and Watanabe, Y. (1972). Vibriocidal antibodies induced by Yersinia enterocolitica serotype IX. Journal of Hygiene, 70, 161-169.

Casewell, M. W., and Phillips, I. (1978a). Epidemiological patterns of klebsiella colonisation and infection in an intensive care unit. Journal of Hygiene, 80, 295-301.
Casewell, M. W., and Phillips, I. (1978b). Food as a source of klebsiella species for colonisation and infection of intensive care patients. Journal of Clinical Pathology, 31, 845-849.

Chambers, A., Kaprove, R. E., Reynolds, W. J., and Urowitz, M. B. (1978). Post-diarrheal arthropathy of Yersinia pseudotuberculosis. Canadian Medical Association Journal, 118, 515-6.

Cowan, S. T., and Steel, K. J. (1965). Manual for the Identification of Medical Bacteria. 1st Edn. Cambridge University Press: Cambridge.

Datta, N. (1969). Drug resistance and $R$ factors in the bowel bacteria of London patients before and after admission to hospital. British Medical Journal, 2, 407-411.

Davies, D. A. L. (1958). The smooth and rough somatic antigens of Pasteurella pseudotuberculosis. Journal of General Microbiology, 18, 118-128.

Davies, D. A. L. (1961). Dideoxysugars of Pasteurella pseudotuberculosis-specific polysacharides/and the occurrence of ascarylose. Nature, 191, 43-44.

Ebringer, A., Cowling, P., Ngwa Suh, N., James, D. C. O., and Ebringer, R. W. (1976). Abstract 1-15. Cross-reactivity between Klebsiella aerogenes species and B27 lymphocyte antigens as an aetiological factor in ankylosing spondylitis. First International Symposium on HLA and Disease. INSERM: Paris.

Ebringer, R. W., Cooke, D., Cawdell, D. R., Cowling, P., and Ebringer, A. (1977). Ankylosing spondylitis: klebsiella and HLA B27. Rheumatology and Rehabilitation, 16, 190-196.

Ebringer, R. W., Cawdell, D. R., Cowling, P., and Ebringer, A. (1978). Sequential studies in ankylosing spondylitis: association of Klebsiella pneumoniae with active disease. Annals of the Rheumatic Diseases, 37, 146-151.

Edwards, P. R., and Ewing, W. H. (1972). Identification of Enterobacterieae. 3rd edn. Burgess Publishing Co: Minneapolis.

Feeley, J. C. (1969). Somatic O antigen relationship of brucella and vibro cholerae. Journal of Bacteriology, 99, 645-649.

Good, A. E., and Schultz, J. S. (1977). Reiter's syndrome following Shigella flexneri 2a. Arthritis and Rheumatism, 20, 100-103.

Hartley, C. L., Clements, H. M., and Linton, K. B. (1977). Escherichia coli in the faecal flora of man. Journal of Applied Bacteriology, 43, 261-269.

Henriksen, S. D. (1954). Studies on the klebsiella group (Kaufman) IV. Cross-reactions of klebsiella types 8, 11, 26, and 35 and the $M$ antigen of Escherichia coli. Acta Patholigica Microbiologica Scandinavica, 34, 271-275.

Hodinka, L., Görnor, B., Merétey, K., Zahmenszky, Z., Geher, P., Telegdy, L., and Bozsóky, S. (1978). HLA-B27 associated spondylarthritis in chronic brucellosis. Lancet, 1, 499.

Hurvell, B., Ahvonen, P., and Thal, E. (1971). Serological cross-reactions between different brucella species and Yersinia enterocolitica. Acta Veterinaria Scandinavica, 12, 86-94.

Kaufman, F. (1949). On the serology of the klebsiella group. Acta Pathologica Microbiologica Scandinavica, Sect. B, 26, 381-406.

Knapp, W. (1968). Serologische Kreuzreactionen Zwischen Pasteurella pseudotuberculosis, Escherichia coli and Enterobacter cloacae. International Symposium on Pseudotuberculosis, Paris 1967, vol. 9, p. 179. Symposium Series on Immunobiological Standardisation. Karger: Basel.

Maeland, J. A., and Digranes, A. (1975). Common enterobacterial antigen in Yersinia enterocolitica. Acta Pathologica Microbiologica Scandinavica, Sect. B, 83, 382-386. 
Montgomerie, J. Z., Doak, P. B., Taylor, D. E. M., North, J. D. K., and Martin, W. J. (1970). Klebsiella in faecal flora of renal transplant patients. Lancet, 2, 787-792.

Orskov, I. (1954). $\mathrm{O}$ antigens in the klebsiella group. Acta Pathologica Microbiologica Scandinavica, Sect. B, 34, 145-156.

Orskov, I., Orskov, F., Jann, B., and Jann, K. (1977). Serology, chemistry and genetics of $O$ and $K$ antigens of Escherichia coli. Bacteriological Reviews, 41, 667-710.

Seldon, R., Lee, S., Wong, W. L. L., Bennet, J. V., and Eickhoff, T. C. (1971). Nosocomial klebsiella infections: intestinal colonisation as a reservoir. Annals of Internal Medicine, 74, 657-664.
Vartainen, J., and Hurri, L. (1964). Arthritis due to Salmonella typhimurium. Acta Medica Scandinavica, 175, 771776.

Warren, C. P. W. (1970). Arthritis associated with salmonella infections. Annals of the Rheumatic Diseases, 29, 483-487.

Wauters, G., Le Minor, L., and Chalon, A. M. (1971). Antigenes somatiques et flagellaires de Yersinia enterocolitica. Annales de l'Institut Pasteur, 120, 631-642.

Winkle, S., Refai, M., and Ronde, R. (1972). On the antigenic relationship of Vibrio cholerae to Enterobacteriaceae. Annales de l'Institut Pasteur, 123, 775-781. 